\title{
PREVALÊNCIA DE ALTERAÇÕES HEPÁTICAS EM BOVINOS ABATIDOS EM MATADOURO-FRIGORÍFICO DO MUNICÍPIO DE PRESIDENTE PRUDENTE, ESTADO DE SÃO PAULO
}

\author{
Raimundo Alberto Tostes ${ }^{1}$ \\ Jorge Minor Fernandes Inagaki ${ }^{2}$ \\ Thiago Chiara Taveira ${ }^{2}$ \\ Hemir Martins Quirilos Assis ${ }^{2}$
}

\begin{abstract}
RESUMO
É fundamental conhecer a prevalência das lesões hepáticas, monitorá-las e assim fornecer material para o trabalho da medicina veterinária preventiva, melhorando o processo de produção e reduzindo perdas econômicas. Neste trabalho foram catalogadas as alterações morfológicas observadas em fígados de bovinos abatidos em um matadouro-frigorífico sob Serviço de Inspeção Federal em Presidente Prudente, São Paulo, durante o período de 01 de abril de 2010 a 31 de março de 2011. As lesões foram catalogadas dentro das seguintes categorias: degenerativas, vasculares, inflamatórias, extra-hepáticas, parasitárias e neoplásicas. Para classificação das alterações, foram consideradas fundamentalmente as características de cor, textura e consistência do parênquima hepático. Foram inspecionados 100.723 animais, sendo $72.638(72,12 \%)$ machos e $28.085(27,88 \%)$ fêmeas. Deste total, 5.204 (5,17\%) exibiam algum tipo de alteração hepática. $\mathrm{O}$ grupo mais significativo de alterações foi de natureza inflamatória, incluindo hepatite, perihepatite e lesões supurativas $(34,69 \%)$. O segundo grupo foi de alterações vasculares, como a telangiectasia, hemorragias capsulares e congestão passiva $(31,80 \%)$. A condenação do fígado por causas extra-hepáticas é alta, estando associada principalmente à contaminação secundária. A fasciolose foi observada em 29 peças de fígado (0,56\%), sugerindo que essa parasitose está adaptada à região, o que chama a atenção pelo seu potencial zoonótico e por ser uma parasitose emergente.
\end{abstract}

Palavras-chave: fígado, inspeção, lesões, matadouro, post mortem.

\section{PREVALENCE OF HEPATIC ALTERATIONS IN CATTLE SLAUGHTERED IN THE CITY OF PRESIDENTE PRUDENTE, SÃO PAULO STATE}

\begin{abstract}
It is fundamental the knowledge about predominance of hepatic lesions, to monitor them and supply material to the preventive veterinary medicine actions, thus improving the production process, reducing economic losses. In this work were catalogued morphological alterations in livers of slaughtered bovines from an abattoir under Federal Inspection Service in Presidente Prudente, São Paulo State, during the period of April 012010 to March 31 2011. The lesions were catalogued according categories: degenerative, vascular, inflammatory, extra hepatic, parasitary and neoplasic. To classify the alterations, was considered fundamentally characteristics like color, texture and hepatic parenchyma consistency. Were inspected 100,723 animals, which 72,638 (72,12\%) males and 28,085 (27,88\%) females. 5,204 (5,17\%) from total showed some kind of hepatic alteration. Most significant alterations group was inflammatory, including hepatitis, perihepatitis and supppurative lesions (34,69\%). Second most representative group was vascular alterations like telangiectasis, capsular hemorrhagies and

${ }^{1}$ Professor Adjunto, Universidade Federal do Paraná.

${ }^{2}$ Universidade Federal do Paraná. Contato principal para correspondência.

Tostes RA, Inagaki JMF, Taveira TC, Assis HMQ. Prevalência de alterações hepáticas em bovinos abatidos em matadouro-frigorífico do município de Presidente Prudente, Estado de São Paulo. Vet. e Zootec. 2017 Set.; 24(3): 604-612.
\end{abstract}


passive congestion $(31,80 \%)$. Liver condemnation by extra-hepatic causes is high, mainly associated to organ secondary contamination. The fasciolosis was observed in 29 parts of liver $(0.56 \%)$, suggesting that this parasitosis is adapted to the region, drawing attention to its zoonotic potential and to being an emerging parasitosis.

Keywords: inspection, lesions, liver, post mortem, slaughterhouse.

\section{PREVALENCIA DE LAS ALTERACIONES HEPÁTICAS EN LOS GANADOS SACRIFICADOS EN MATADERO DE LA CIUDAD DE PRESIDENTE PRUDENTE, ESTADO DE SÃO PAULO}

\section{RESUMEN}

Es fundamental conocer la prevalencia de las lesiones hepáticas, monitorearlas y con este proporcionar material para el trabajo de la medicina veterinaria preventiva, mejorando el proceso de la producción y reduciendo pierdas económicas. En este trabajo fueron catalogadas las alteraciones morfológicas observado en hígado de los ganados sacrificados en uno matadero con servicio de inspección federal en Presidente Prudente, São Paulo, durante lo período de 01 de abril de 2010 a 31 de marzo de 2011. Las lesiones fueron catalogadas entre las siguientes categorías: degenerativas, vasculares, inflamatorias, extra-hepáticas, parasitarias y neoplásicas. Para la clasificación de las alteraciones, fueron consideradas fundamentalmente las características del color, textura y consistencia del parénquima hepático. Fueron inspeccionados 100.723 animales, siendo $72.638(72,12 \%)$ machos y $28.085(27,88 \%)$ hembras. De este total $5.204(5,17 \%)$ he exhibido alguna alteración hepática. El grupo más significativo de alteración fue de la naturaleza inflamatoria, incluyendo hepatitis, perihepatitis y lesiones supurativas $(34,69 \%)$. El según grupo fue de las alteraciones vasculares como la telangiectasia, hemorragias capsulares y congestión pasiva $(31,80 \%)$. La condenación del hígado debido causas extrahepáticas es alta, estando asociada principalmente a la contaminación secundaria. La fasciolosis se observó en 29 trozos de hígado (0.56\%), lo que sugiere que este parásito estás adaptado en la región, que llama la atención por su potencial zoonótico y por ser una enfermedad parasitaria emergente.

Palabras clave: hígado, inspección, lesiones, matadero, post mortem.

\section{INTRODUÇÃO}

O Brasil é um dos principais produtores e exportadores de carne bovina no mundo. Segundo dados do IBGE, em 2011 o rebanho brasileiro teve um efetivo de 212,798 milhões de cabeças, sendo que somente no último trimestre foram abatidos cerca de 7,36 milhões de cabeças de bovinos em todo país, dentre as quais o Estado de São Paulo foi responsável por 809.898 delas (1,2). Destaca-se que só a microrregião de Presidente Prudente, em 2011, possuía um efetivo de aproximadamente 1.547 .941 cabeças, $14 \%$ do total do rebanho paulista (2).

$\mathrm{O}$ acesso às informações relativas à prevalência de várias doenças e lesões observadas no abate, incluindo as alterações hepáticas de naturezas diversas, é restrito. Estes dados normalmente só estão disponíveis internamente aos serviços de inspeção sanitária.

Vários estudos têm situado a frequência de alterações hepáticas em bovinos ao abate (36). Estas alterações constituem um grupo importante para saúde humana e também para os matadouros-frigoríficos sob o ponto de vista comercial. Não há uma estimativa precisa das perdas econômicas decorrentes de condenação de vísceras no Brasil, mas acredita-se que, em algumas regiões, podem ocorrer prejuízos significativos para a pecuária. 
O fígado bovino está localizado quase na sua totalidade à direita do plano medial, seu peso médio é de 4,5 a $5,5 \mathrm{~kg}$ em um bovino adulto $(7,8)$ correspondendo, segundo Dyce et al. (9), de $1 \%$ a $1,5 \%$ do peso corpóreo. Segundo Gürtler (10), no bovino, a relação existente entre a massa corpórea e a massa hepática é de 1:55.

As principais alterações que motivam a condenação do fígado de bovinos em matadouros são as de natureza inflamatória (11), sendo o abscesso hepático, que ocorre com mais frequência em bovinos de engorda e observado em animais aparentemente saudáveis, a mais importante. A ocorrência varia de $1 \%$ a $95 \%$ em alguns lotes $(5,12)$. Contudo, várias outras alterações aparecem como causa de condenação desse órgão.

Outra alteração de natureza inflamatória de impacto econômico e sanitário é a tuberculose. Duas formas fundamentais de tuberculose são encontradas no fígado: a forma miliar, caracterizada por nódulos pequenos, bem delimitados, de cor branco-amarelada e com centros turvos, quando há calcificação, disseminados no parênquima adjacente do órgão; e a forma nodular, com lesões maiores solitárias ou múltiplas, com volume variando entre uma noz e um punho de um indivíduo adulto. Em seu interior encontra-se a típica caseificação e em alguns casos há na lesão presença de matéria calcária apresentando como característica o ranger da faca ao corte (11).

Alterações degenerativas são igualmente importantes em fígados bovinos, destacando-se como a principal, neste caso, a esteatose hepática (13). Trata-se de um acúmulo de lipídeos no parênquima do órgão e está associado a causas tóxicas e anóxicas.

Dentre as alterações vasculares, a telangiectasia ocupa o lugar mais importante como causa de condenação do fígado à inspeção. É caracterizada por pequenas e múltiplas manchas de coloração avermelhada ou púrpura, vistas tanto na superfície capsular como no parênquima do órgão, ao corte. Histologicamente, trata-se de uma dilatação acentuada de sinusóides em áreas que os hepatócitos foram perdidos (14-16).

Entre as alterações hepáticas de origem parasitária mais significativa, a principal é a fasciolose, que é provocada pelo trematódeo Fasciola hepatica, seguida pela hidatidose, que no bovino pode aparecer de forma multifocal e tem como agente o cestóide Echinococcus granulosus, embora com pouco significado clínico e patológico (17).

É incomum a ocorrência de neoplasias primárias no fígado bovino, mas, dentre os achados anatomopatológicos possíveis de serem detectados na linha de abate, estão o carcinoma hepatocelular, o colangiocarcinoma e os tumores vasculares - hemangioma e hemangiossarcoma (11). O envolvimento secundário do fígado por neoplasias pode ser visto principalmente na leucose e em outras neoplasias metastáticas da cavidade abdominal (11).

A caracterização das alterações hepáticas em bovinos e o estabelecimento de um diagnóstico preciso no frigorífico é uma tarefa complexa, uma vez que muitas delas ocorrem simultaneamente em mais de um sistema orgânico.

Segundo o Regulamento da Inspeção Industrial e Sanitária de Produtos de Origem Animal (RIISPOA) (18), a inspeção da carcaça e dos órgãos é realizada de forma a verificar não somente a lesão no órgão em questão, mas também o comprometimento do restante da carcaça e demais órgãos. Lesões de cisticercose devem ser avaliadas conforme a característica, quantidade e a afecção do restante da carcaça, podendo levar a condenação parcial ou total da carcaça e do órgão. Fígados com fasciolose, tuberculose, cirrose, hepatites, tumores, aderências, coloração anormal, congestão e hemorragias são sempre condenados em sua totalidade (18).

O conhecimento da prevalência das alterações hepáticas é fundamental para que haja um melhor preparo dos médicos veterinários e seus auxiliares no monitoramento e identificação das lesões no abate, promovendo a redução das perdas econômicas e a melhora do processo produtivo. Adicionalmente, conhecer esses dados pode facilitar a implementação de programas preventivos no campo. Portanto, o objetivo do presente trabalho foi avaliar a prevalência das

Tostes RA, Inagaki JMF, Taveira TC, Assis HMQ. Prevalência de alterações hepáticas em bovinos abatidos em matadouro-frigorífico do município de Presidente Prudente, Estado de São Paulo. Vet. e Zootec. 2017 Set.; 24(3): 604-612. 
principais alterações hepáticas responsáveis pela condenação do órgão realizadas por fiscais do Serviço de Inspeção Federal (S.I.F) e também as perdas econômicas decorrentes.

\section{MATERIAL E MÉTODOS}

Foram catalogadas as alterações morfológicas presentes em fígados de bovinos abatidos em um matadouro-frigorífico localizado na região de Presidente Prudente, Estado de São Paulo. A observação das alterações morfológicas foi feita mediante o acompanhamento das atividades do S.I.F. durante o período de 01 de abril de 2010 a 31 de março de 2011.

No total foram inspecionados 100.723 animais, dos quais $72.638(72,12 \%)$ eram machos e $28.085(27,88 \%)$ eram fêmeas.

As lesões foram catalogadas a partir das triagens efetuadas pelo S.I.F. O fígado era examinado na linha de inspeção específica (linha E) de acordo com o procedimento padrão preconizado pelo S.I.F. A partir da identificação de qualquer anormalidade, o órgão era separado para o Departamento de Inspeção Final (D.I.F.) para avaliação da natureza e extensão da alteração presente.

As lesões foram catalogadas dentro das seguintes categorias: degenerativas, vasculares, inflamatórias, extra-hepáticas, parasitárias e neoplásicas.

$\mathrm{Na}$ análise das alterações foram consideradas fundamentalmente as características de cor, textura e consistência do parênquima hepático.

$\mathrm{Na}$ presença de alterações parasitárias, amostras dos espécimes encontrados foram colhidas em solução de álcool a $70 \%$ e encaminhados para identificação parasitológica no laboratório de Parasitologia Veterinária da Universidade do Oeste Paulista.

A fim de estabelecer um cálculo para o prejuízo causado pela condenação do fígado, foi usado como base o valor de $\mathrm{R} \$ 7,30$ por quilograma de fígado, preço médio praticado no varejo da cidade de São Paulo no período de abril de 2010 a março de 2011 (19). Para fins de cálculo, o peso médio do fígado de um bovino adulto foi estimado em $5,0 \mathrm{~kg}$, conforme adotado por Getty (7). Desta forma, estimou-se uma perda de $\mathrm{R} \$ 36,50$ por órgão hepático condenado, equivalente a U\$S 21,09 por órgão ou U\$S 4,22 por quilograma de fígado no período em que as amostras foram coletadas ${ }^{1}$.

\section{RESULTADOS E DISCUSSÃO}

Nas últimas décadas, tem sido crescente a preocupação em aperfeiçoar a produção animal, incrementando a seleção genética, pesquisando a nutrição animal, avaliando pontos críticos dentro da cadeia produtiva e a sanidade animal. Essa valorização também se traduz na pesquisa e na investigação de realidades regionais muitas vezes desconhecidas para os médicos veterinários. Um exemplo disto é o desconhecimento sobre a prevalência de lesões em vísceras de bovinos abatidos para consumo no Brasil.

Neste estudo, do total de animais inspecionados, 5.204 animais $(5,17 \%)$ exibiam algum tipo de alteração hepática.

Na tabela 1 são apresentadas as categorias de alterações encontradas e o seu percentual em relação ao total examinado. As alterações encontradas variaram significativamente em intensidade, frequência de aparecimento e apresentação morfológica. Não foi possível caracterizar de forma individualizada lesões como hemorragias capsulares e parenquimais, lacerações, congestão aguda e congestão passiva crônica. Todas estas alterações foram genericamente agrupadas como alterações vasculares.

\footnotetext{
${ }^{1}$ Considerado o valor médio do dólar americano ( $\left.\mathrm{R} \$ 1,00=\mathrm{U} \$ \mathrm{~S} 1,73\right)$ no período de abril/2010-março/2011 segundo a variação cambial (http://portalibre.fgv.br/).

Tostes RA, Inagaki JMF, Taveira TC, Assis HMQ. Prevalência de alterações hepáticas em bovinos abatidos em matadouro-frigorífico do município de Presidente Prudente, Estado de São Paulo. Vet. e Zootec. 2017 Set.; 24(3): 604-612.
} 
Tabela 1. Percentual de fígados acometidos, num total de 100.723 bovinos abatidos, por categoria de alterações.

\begin{tabular}{ccc}
\hline Categoria de Alteração & $\begin{array}{c}\text { Número de } \\
\text { Fígados Afetados }\end{array}$ & $\begin{array}{c}\text { \% em relação ao total } \\
\text { examinado }\end{array}$ \\
\hline Alterações Inflamatórias & 1.805 & 34,69 \\
Alterações Vasculares & 1.655 & 31,80 \\
Alterações Extra-Hepáticas & 1.569 & 30,15 \\
Alterações Parasitárias & 147 & 2,82 \\
Alterações Neoplásicas & 21 & 0,40 \\
Alterações Degenerativas & 7 & 0,14 \\
\hline Total & 5.204 & 100,0 \\
\hline
\end{tabular}

No presente estudo o grupo mais significativo de alterações foi das lesões de natureza inflamatória, incluindo hepatite e lesões supurativas. O segundo grupo foi o de alterações vasculares, sendo que neste grupo foram incluídas lesões como a telangiectasia, hemorragias capsulares e congestão passiva.

O predomínio das alterações hepáticas de natureza inflamatória neste estudo é concordante com Moreira (20) e Mello et al. (21). Contudo, são diferentes daqueles observados por Lauzer et al. (3). Moreira (20) acrescenta que o prejuízo devido à condenação do fígado de bovinos para graxaria é cerca de quatro vezes superior ao da condenação das carcaças correspondentes.

Com relação às lesões hepáticas supurativas é interessante observar que o espectro etiológico é bastante variável. Segundo Nagaraja e Chengappa (12), os abscessos hepáticos em gado de corte parecem estar frequentemente associados à dieta e ao manejo alimentar. Além da condenação parcial ou total do fígado na inspeção, os prejuízos causados por essas lesões incluem a diminuição do apetite dos animais acometidos, piora na conversão alimentar e um rendimento de carcaça menor.

A gênese dos abscessos parece ser uma ruminite, que na maioria dos casos é provocada pelo Fusobacterium necrophorum. Essa bactéria possui ou produz fatores de virulência que participam na introdução e colonização do epitélio ruminal, com a subsequente invasão e colonização do fígado. Narayanan et al. (22) isolaram amostras de F. necrophorum de bovinos que exibiam abscessos hepáticos e ruminite necrótica e comprovaram que as amostras eram geneticamente idênticas em ambas as lesões. A mesma comprovação foi feita com relação à Arcanobacterium (Actinomyces) pyogenes por Narayanan et al. (23).

Outra alteração inflamatória significativa é a tuberculose (TB). No presente estudo, $1,25 \%$ das lesões observadas foram atribuídas à TB. Lauzer et al. (3), no Brasil, verificaram uma prevalência de $0,1 \%$ de TB com envolvimento hepático, enquanto Milian-Suazo et al. (24) verificaram uma prevalência de $16 \%$ de TB com envolvimento hepático no México. Contudo, Corner et al. (25) alertam para o fato de que a inspeção em matadouros falha em um número significativo de casos de animais positivos para TB. A explicação para este fato provavelmente decorre da presença de lesões tuberculosas solitárias ou isoladas e cujo diagnóstico de TB é negligenciado ou subestimado. Corner et al. (25) comprovaram haver a falha no reconhecimento de até $47 \%$ dos animais positivos para tuberculose.

Isoladamente, as lesões extra-hepáticas foram responsáveis pela maior parte das condenações do fígado (Tabela 1). Entre estas alterações estão incluídos, principalmente, os processos de natureza inflamatória e que contaminam o fígado, quer isso ocorra de forma embólica ante-mortem ou post-mortem na evisceração.

A telangiectasia foi a segunda alteração hepática mais prevalente (Tabela 2). Destaca-se por apresentar prevalência significativamente maior do que o observado em estudos correlatos que situam esta lesão entre $1,3 \%$ e $10 \%(15,16,26)$. De acordo com o RIISPOA, quando as 
lesões comprometerem mais da metade do órgão, este deve ser condenado totalmente e quando em menor quantidade, deve ser submetido a aproveitamento condicional (18).

As alterações de origem parasitária corresponderam a 2,82\% dos fígados afetados (Tabela 1). Dentre as alterações parasitárias presentes houve a identificação de quatro casos de larvas de Cysticercus bovis, 121 casos de larvas de Echinococcus granulosus e 29 casos de Fasciola hepatica (Tabela 2).

Embora as lesões parasitárias tenham aparecido em pequeno número, foi importante notar, principalmente, o aparecimento de casos de fasciolose. Tostes et al. (27) diagnosticaram a ocorrência do primeiro caso autóctone de Fasciola hepatica na região de Presidente Prudente, com indícios consistentes da presença e manutenção do ciclo do parasita na propriedade estudada. A ocorrência destas lesões, por se tratar de uma espécie zoonótica, leva a condenações do órgão afetado e até a rejeição total da carcaça no caso de infecções associadas a outros quadros, como a icterícia.

A tabela 2 apresenta, de forma sumária, o percentual de fígados afetados, de acordo com o diagnóstico macroscópico realizado no frigorífico.

Tabela 2 - Percentual de fígados acometidos de acordo com o diagnóstico macroscópico.

\begin{tabular}{ccc}
\hline Alteração & $\begin{array}{c}\text { Número de } \\
\text { Fígados Afetados }\end{array}$ & $\begin{array}{c}\text { \% em relação ao total } \\
\text { examinado }\end{array}$ \\
\hline Extra-hepática & 1.569 & 30,15 \\
Telangiectasia & 1.112 & 21,37 \\
Abcesso & 770 & 14,80 \\
Congestão & 543 & 10,43 \\
Perihepatite & 535 & 10,28 \\
Contaminação & 423 & 8,13 \\
Hidatidose & 114 & 2,19 \\
Tuberculose & 65 & 1,25 \\
Fasciolose & 29 & 0,56 \\
Neoplasia & 21 & 0,40 \\
Cirrose & 11 & 0,21 \\
Degeneração & 7 & 0,13 \\
Cisticercose & 4 & 0,08 \\
Hepatite & 1 & 0,02 \\
\hline Total & 5.204 & 100,0 \\
\hline
\end{tabular}

Também é importante reforçar que as perdas econômicas decorrentes da condenação do fígado atingem cifras altas, acarretando em prejuízos para produtores e frigoríficos e levando à oferta menor do produto ao mercado.

No intervalo de um ano, a perda com a condenação do fígado no frigorífico atingiu aproximadamente $26.020 \mathrm{~kg}$. A estimativa de perda econômica com a condenação do fígado foi de R\$ 15.828,83 ou U\$S 9.149,62 ao mês, ainda R\$ 189.946,00 ou U\$S 109.795,37 ao ano no preço de varejo. A despeito de este número ser modesto em âmbito local, é preciso considerar que muitas lesões hepáticas são uma repercussão de lesões sistêmicas e que aumentam este valor.

Para os frigoríficos, a perda por contaminação foi de $\mathrm{R} \$ 15.439,50$ e merece destaque, por se tratar a de uma falha de ordem tecnológica, que poderia ser prevenida com aplicações de medidas corretivas no estabelecimento, ao contrário das demais, que são na maioria multifatoriais, cujas intervenções caberiam ao manejo no campo e aos cuidados com a sanidade do rebanho.

Contudo, a realização dos trabalhos de inspeção post-mortem tem como principal motivação eliminar do consumo alimentos potencialmente danosos à saúde pública, o que 
acarretaria custos ainda maiores com os tratamentos dos enfermos. Deste modo, a inspeção não deve ser julgada como um fator gerador de prejuízos, mas como uma ferramenta de prevenção a danos ainda maiores. Cabe à indústria, em conjunto com criadores e médicos veterinários, estudar a viabilidade e o custo da implementação de programas de prevenção no campo, visando a redução de condenações no frigorífico.

De um modo geral, foi verificada a concordância dos achados com as alterações referidas na literatura, reforçando a necessidade de identificar pontos críticos na cadeia de produção de gado de corte a fim de minimizar a condenação do fígado, de outras vísceras e também da carcaça no momento do abate.

\section{CONCLUSÃO}

Das causas que levam à rejeição do fígado predominam as lesões de natureza inflamatória, principalmente na forma de abscessos. E a condenação do fígado por causas extrahepáticas é alta, estando associada principalmente à contaminação secundária do órgão. Perdas do fígado no município de Presidente Prudente atingem valores relevantes. A fasciolose, embora aparecendo em pequeno número, chamou a atenção por tratar-se de parasitose emergente e provavelmente adaptada às condições geoclimáticas da região.

\section{REFERÊNCIAS}

1. Instituto Brasileiro Geografia e Estatística. Produção pecuária municipal 2011. Rio de Janeiro: IBGE; 2011.

2. Instituto Brasileiro de Geografia e Estatística. Banco de dados agregados [Internet]. Rio de Janeiro: IBGE; 2013 [cited 2013 Apr 4]. Available from: www.sidra.ibge.gov.br

3. Lauzer JJ, Silva SF, Costa DF, Santos AF. Condenações de fígados de bovinos em Santa Maria, RS. Rev Cent Cienc Rurais. 1979;9:251-6.

4. Moreira EC, Baptista F. Causas de condenação de fígados bovinos em frigoríficos de Minas Gerais e perdas econômicas associadas. Hig Aliment. 1999;62(13):22-7.

5. Mendes RE, Pilati C. Estudo morfológico de fígado de bovinos abatidos em frigoríficos industriais sob inspeção estadual no Oeste e no Planalto de Santa Catarina, Brasil. Cienc Rural. 2007;37(6):1728-34.

6. Vieira NP, Faria PB, Mattos MR, Pereira AA. Condenação de fígados bovinos na região sul do estado do Espírito Santo. Arq Bras Med Vet Zootec. 2011;63(6):1605-8.

7. Getty R. Sistema digestivo. In: Getty R. Anatomia dos animais domésticos. 5a ed. Rio de Janeiro: Guanabara-Koogan; 1986. v.2, p.1188-202.

8. König HE, Liebich HG. Anatomia dos animais domésticos. Órgãos e sistemas. Porto Alegre: Artmed; 2004. v.2.

9. Dyce KM, Sack WO, Wensing CJG. Aparelho digestório. In: Dyce KM, Sack WO, Wensing CJG. Tratado de anatomia veterinária. 3a ed. Rio de Janeiro: Elsevier; 2004. p.99-145. 
10. Gürtler H. Fisiologia do fígado. In: Gürtler H. Fisiologia veterinária. 4a ed. Rio de Janeiro: Guanabara-Koogan; 1987. p.341-7.

11. Prata LF, Fukuda RT. Fundamentos de higiene e inspeção de carnes. 1a ed. Jaboticabal: Funep; 2001.

12. Nagaraja TG, Chengappa MM. Liver abscesses in feedlot cattle: a review. J Anim Sci. 1998;76(1):287-98.

13. Raoofi A, Bazargani TT, Tabatabayi AH. An abattoir survey on the frequency of fatty liver syndrome in dairy cows from the suburbs of Tehran. Trop Anim Health Prod. 2001;33(1):15-9.

14. MacLachlan NJ, Cullen JM. Fígado, sistema biliar e pâncreas exócrino. In: Carlton WW, McGavin MD. Patologia veterinária especial de Thomson. 2a ed. Porto Alegre: Artmed; 1998. p.95-131.

15. Stotland EI, Edwards JF, Roussel AJ, Simpson RB. Bacterial microflora of normal and telangiectatic livers in cattle. J Am Vet Med Assoc. 2001;219(1):36-9.

16. Atasever A, Vural SA, Berkin S. Incidence and pathologic studies on liver telangiectasis in beef cattle. Turk J Vet Anim Sci. 2002;26:235-8.

17. Urquhart GM. Parasitologia Veterinária. 2a ed. Rio de Janeiro: Guanabara-Koogan; 1998.

18. Decreto $\mathrm{n}^{\circ} 30.691$, de 29 Março de 1952. Regulamento da inspeção industrial e sanitária de produtos de origem animal. Diário Oficial da União. 7 Jul 1952. p.10.785.

19. Instituto de Economia Agrícola do Estado de São Paulo. Levantamento dos preços médios no varejo da cidade de São Paulo [Internet]. São Paulo: IEA; 2012 [cited 2012 Aug 22]. Available from: http://ciagri.iea.sp.gov.br/nia1/precos_medios.aspx?cod_sis=4.

20. Moreira EC. Importância do controle da sanidade sobre produtos de origem animal [Internet]. São Paulo; 2001 [cited 2013 Mar 27]. Available from: http://www.simcorte.com/index/Palestras/s_simcorte/10_elvio.PDF

21. Mello FAM, Fernandez AT, Machado TCC, Frederico FR, Oliveira AJ. Ocorrência de condenações de órgãos comestíveis de bovinos, em matadouros sob regimes de inspeção estadual e federal no Estado do Rio de Janeiro, RJ. Hig Aliment. 2005;19(137):56-62.

22. Narayanan S, Nagaraja TG, Okwumabua O, Staats J, Chengappa MM, Oberst RD. Ribotyping to compare Fusobacterium necrophorum isolates from bovine liver abscesses, ruminal walls, and ruminal contents. Appl Environ Microbiol. 1997;63(12):4671-8.

23. Narayanan S, Nagaraja TG, Wallace N, Staats J, Chengappa MM, Oberst RD. Biochemical and ribotypic comparison of Actinomyces pyogenes and A pyogenes-like organisms from liver abscesses, ruminal wall, and ruminal contents of cattle. Am $J$ Vet Res. 1998;59(3):271-6. 
24. Milian-Suazo F, Salman MD, Ramirez C, Payeur JB, Rhyan JC, Santillan M. Identification of tuberculosis in cattle slaughtered in Mexico. Am J Vet Res. 2000;61(1):86-9.

25. Corner L, Melville L, McCubbin K, Small KJ, McCormick BS, Wood PR, et al. Efficiency of inspection procedures for the detection of tuberculous lesions in cattle. Aust Vet J. 1990;67(6):389-92.

26. Julian LM. Studies on the subgross anatomy of the bovine liver II. The pathology of telangiectasis as demonstrated by the vinylite-corrosion technique. Am J Vet Res. 1950;11(39):166-72.

27. Tostes RA, Santarém VA, Alberti H, Sanches OC. Casos autóctones de Fasciola hepática na região de Presidente Prudente, São Paulo, Brasil. Cienc Rural. 2004;34:961-2.

Recebido em: 03/08/2016

Aceito em: 11/08/2017

Tostes RA, Inagaki JMF, Taveira TC, Assis HMQ. Prevalência de alterações hepáticas em bovinos abatidos em matadouro-frigorífico do município de Presidente Prudente, Estado de São Paulo. Vet. e Zootec. 2017 Set.; 24(3): 604-612. 